\title{
THE BLACK HOLE GRAZER
}

\section{A NEW BINARY BLACK HOLE ENGINE IN ACTIVE GALACTIC NUCLEI}

\author{
Y. TANIGUCHI \& O. KABURAKI \\ Astronomical Institute, Tohoku University \\ Aramaki, Aoba, Sendai 980-77, JAPAN
}

\section{THE BLACK HOLE GRAZER}

We propose an alternative model for the powering of active galactic nuclei (AGN), based on the assumption that all AGN have experienced mergers. In our model (Kaburaki and Taniguchi 1996; Taniguchi and Kaburaki 1996), a close pair of super-massive black holes (the black hole grazer) orbit one another in a plane roughly perpendicular to the galactic center magnetic field. The orbital motion induces surface charges on the black holes which produce an electric field. This field is strong enough to cause pair creation so that the Roche lobe of the binary system is filled with pair plasmas. Rigid-body rotation of the Roche-lobe magnetosphere drives electrodynamically a powerful synchrotron jet emanating from the center of mass of the binary. Furthermore, a pair of equatorial jets flow from the outer Lagrangian points of the binary system. Although these jets are not so collimated, they interact with the accreting gas ring formed around the orbital plane of the binary, causing broad line regions or $\mathrm{H}_{2} \mathrm{O}$ maser emission regions (Taniguchi et al. 1996). In addition to the primary jet, two secondary jets are also driven by local accretion disks around the two black holes. The interaction among the primary and the secondary jets may explain detailed jet morphology observed by VLBI facilities.

\section{References}

Kaburaki, O., and Taniguchi, Y. (1996) Binary Black-Hole Engines in Active Galactic Nuclei, Physics of Accretion Disks: Advection, Radiation, and Magnetic Field, edited by S. Kato et al. (Gordon and Breach Science Publishers), 327

Taniguchi, Y., and Kaburaki, O. (1996) A New Unified Model of Active Galactic Nuclei, The Physics of LINERs in View of Recent Observations, ASP Conf. Ser. 103, 227

Taniguchi, Y., Kaburaki, O., Ohyama, Y., and Murayama, T. (1996b) A Supermassive Black Hole Binary in NGC 4258, The Physics of LINERs in View of Recent Observations, ASP Conf. Ser. 103, 221 


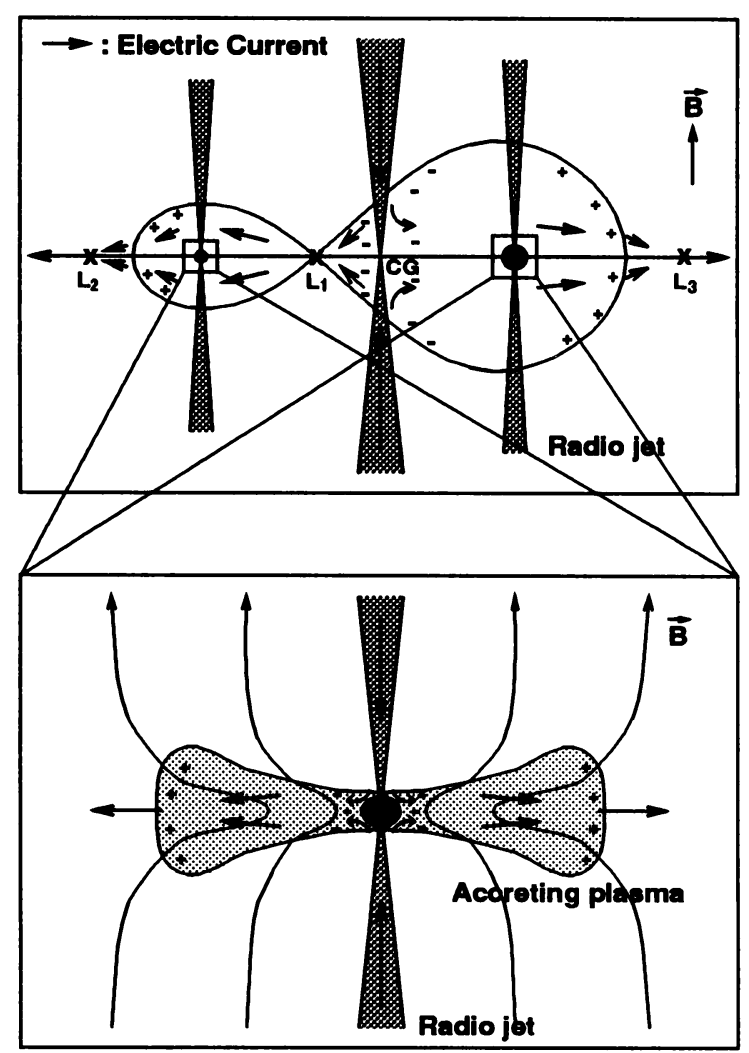

Figure 1. The grazing model of the super massive black-hole binary system. [1] (upper panel) The primary jets emanating from the center of gravity (CG) of the binary system. When the two super massive black holes graze near the pericenter, the two black holes emit intense electric dipole emission. The dipole emission introduces a large-scale electric field around the black holes, resulting electron-positron pair creation. The rotation of the plasma filling the Roche lobe drives an outward electric current from CG. [2] (lower panel) The two secondary jets emanating from the two black holes. The rotation of accreting plasma disks formed around the two black holes drives similar systems of electric current. The physical mechanism of the jets is the same as that of the primary jets. Each vertical pair of return current along the magnetic field grows into a highly collimated, bipolar radio jets. 